\title{
Association between Relative Handgrip Strength and Dyslipidemia in Korean Adults: Findings of the 2014-2015 Korea National Health and Nutrition Examination Survey
}

\author{
Bo Mi Kim ${ }^{1,2,3}$, Yu Hyeon $\mathrm{Yi}^{1,2,3,4, *}$, Yun Jin Kim ${ }^{1,2,3}$, Sang Yeoup Lee ${ }^{5,6}$, Jeong Gyu Lee ${ }^{1,2,3,4}$, Young Hye Cho ${ }^{5}$, \\ Young Jin Tak ${ }^{1,2,3}$, Hye Rim Hwang ${ }^{1,2,3}$, Seung Hun Lee ${ }^{1,2,3,4}$, Eun Ju Park ${ }^{5}$, Youngin Lee ${ }^{4,5}$ \\ 'Department of Family Medicine, Pusan National University School of Medicine, Yangsan, Korea \\ ${ }^{2}$ Pusan National University Medical Research Institute, Yangsan, Korea \\ ${ }^{3}$ Department of Family Medicine, Pusan National University Hospital, Busan, Korea \\ ${ }^{4}$ Busan Tobacco Control Center, Pusan National University Hospital, Busan, Korea \\ ${ }^{5}$ Family Medicine Clinic, Obesity, Metabolism and Nutrition Center and Research Institute of Convergence of Biomedical Science and Technology, \\ Pusan National University Yangsan Hospital, Yangsan, Korea \\ ${ }^{6}$ Department of Medical Education, Pusan National University School of Medicine, Yangsan, Korea
}

Background: Grip strength is a convenient method to measure muscle strength. Recently, relative handgrip strength (HGS) was recommended as a clinical predictor of metabolic health and disease, such as dyslipidemia, which is considered a risk factor for cardiovascular disease. The purpose of this study was to characterize the association between relative HGS and dyslipidemia.

Methods: We included 6,027 adults (2,934 men, 3,093 women) aged 30-69 years who participated in the Korea National Health and Nutrition Examination Survey in 2014 and 2015. Relative HGS was obtained by dividing the HGS by body mass index. Complex sampling analysis was conducted to compare the general characteristics of participants according to the quartiles of relative HGS. Logistic regression analysis was used to examine the association between quartiles of relative HGS and dyslipidemia.

Results: After adjustment for age, prevalence of diabetes mellitus, prevalence of hypertension, alcohol consumption, smoking status, exercise, income, and education level, relative HGS was inversely associated with dyslipidemia in both men and women. In multivariable logistic regression analysis, the odds ratios (95\% confidence intervals) for dyslipidemia in quartiles 1,2 , and 3 relative to quartile 4 were 1.36 (1.00-1.83), 1.29 (0.98-1.70), 1.23 (0.95$1.60)$ in men and 1.81 (1.30-2.50), 1.81 (1.32-2.47), 1.39 (1.07-1.81) in women, respectively.

Conclusion: Relative HGS was inversely associated with dyslipidemia risk in Korean adults. Muscle-strengthening exercise is recommended to enhance health outcomes.

Keywords: Hand Strength; Muscle Strength; Dyslipidemias; Metabolic Syndrome

Received: June 18, 2019, Revised: September 26, 2019, Accepted: October 4, 2019

*Corresponding Author: Yu Hyeon Yi https://orcid.org/0000-0002-1786-2737

Tel: +82-51-240-7834, Fax: +82-51-240-7843, E-mail: eeugus@hanmail.net 


\section{INTRODUCTION}

Cardiovascular deaths account for $31 \%$ of annual global deaths. ${ }^{1)}$ In Korea, cardiovascular disease was the second leading cause of death in 2016. ${ }^{2)}$ Dyslipidemia is a major risk factor for cardiovascular disease. The prevalence of dyslipidemia in Korean adults aged 30 years or older was $47.8 \%$ ( $57.6 \%$ for men and $38.3 \%$ for women) in 2013 , and the prevalence increases with age. ${ }^{3)}$ The most common risk factors for cardiovascular disease in men were hypertension, smoking, dyslipidemia, and diabetes, and the combined risk for cardiovascular disease in the presence of all four risk factors was $64 \%$. The most common risk factors for cardiovascular disease in women were hypertension, dyslipidemia, diabetes, and smoking. ${ }^{4)}$ For these reasons, dyslipidemia is a common and important health problem from the perspective of cardiovascular disease prevention in Korea.

Handgrip strength (HGS) is a useful predictor of muscle mass and strength that is convenient and simple to measure. ${ }^{5,6)}$ In several studies, low HGS has been associated with metabolic diseases, cardiovascular disease, and mortality. ${ }^{7-9)}$ One study reported that sarcopenia was associated with the presence of cardiovascular disease independent of other cardiovascular risk factors after adjusting for renal function and medications in Korean elders. ${ }^{10)}$ There is also evidence that grip strength is inversely associated with all-cause mortality, cardiovascular mortality, and cardiovascular disease. ${ }^{11)}$ The relationship between grip strength and the metabolic syndrome has also been examined in many studies, with conflicting results. In a cross-sectional study of 2,677 adults in the United Kingdom, lower HGS was associated with higher triglycerides (TG), blood pressure (BP), and insulin resistance after adjusting for age, weight, and health behavior. ${ }^{9)}$ In a cross-sectional study of 5,014 Korean adults, the group with metabolic syndrome had a higher HGS than the group without metabolic syndrome. ${ }^{12)}$

Grip strength is known to be affected by weight, height, and body mass, and has a positive correlation with body mass index (BMI). ${ }^{13)}$ There is no standardized grip strength index, but previous studies have used relative HGS, which is calculated as absolute HGS divided by BMI. In recent studies on muscle health, the use of relative HGS adjusted for BMI has been recommended. Relative to absolute HGS, relative HGS has stronger associations with metabolic syndrome and its component parameters, including high-density lipoprotein cholesterol (HDLC), TG, fasting glucose, and $\mathrm{BP}^{12)}$ In addition, previous reports have shown that relative HGS is negatively associated with cardiometabolic risk ${ }^{14,15)}$ and predicts cardiovascular and all-cause mortality among middle-aged and elderly people. ${ }^{16)}$

This study aimed to evaluate the association between relative HGS and dyslipidemia, and to investigate the hypothesis that low relative HGS is associated with dyslipidemia.

\section{METHODS}

\section{Study Population}

We analyzed data from the second and third years of the Korea Na- tional Health and Nutrition Examination Survey (KNHANES) VI (2014-2015). The KNHANES was performed to examine the health and nutritional status of the Korean population. The survey used data from complex, nationwide, stratified, multistage probability sampling to represent the Korea population. Data on participants' demographic characteristics, health behavior, and health examinations were obtained by the Korea Centers for Disease Control and Prevention (KCDC) annually. The KNHANES protocol, following the Declaration of Helsinki, was reviewed and approved by the institutional review board of the KCDC (2013-12EXP-03-5, 2015-01-02-6C). Written informed consent for individual data was obtained from all participants.

Of the individuals aged $\geq 19$ years who participated in the 2014-2015 KNHANES $(n=11,921)$, those aged $30-69$ years were included $(n=$ 8,361 ). Those who had missing data, including missing survey entries on the treatment of dyslipidemia, level of lipids, HGS measurement, and BMI, were excluded ( $n=2,334)$. Altogether, 6,027 participants were ultimately included in this study.

\section{Measurement of Handgrip Strength}

A digital grip strength dynamometer (Model TKK 5401; Takei Scientific Instruments Co. Ltd., Tokyo, Japan) was used to measure grip strength. The grip strength measurement was performed in a standing position with the arm extended. The middle finger was held at $90^{\circ}$ to the handle and measured while exhaling. The maximal hold time of the dynamometer was 3 seconds, and each hand was tested 3 times. Absolute HGS was calculated as the average of the three measurements of the dominant hand. Relative HGS was defined as the absolute HGS divided by BMI.

\section{Definition of Dyslipidemia}

A health questionnaire confirmed the use of medication for dyslipidemia. After at least 8 hours of fasting, blood samples were collected and analyzed within 24 hours. The Hitachi Automatic Analyzer 7600210 (Hitachi, Tokyo, Japan) was used to measure total cholesterol (TC), HDLC, TG, and low-density lipoprotein cholesterol (LDLC) levels. According to the " 2015 Korean guidelines for management of dyslipidemia," published by the Korean Society of Lipidology and Atherosclerosis, dyslipidemia was defined as having one of the following criteria: currently under medication for dyslipidemia, TC $\geq 240 \mathrm{mg} / \mathrm{dL}$, HDLC $<40 \mathrm{mg} / \mathrm{dL}, \mathrm{TG} \geq 200 \mathrm{mg} / \mathrm{dL}$, and LDLC $\geq 160 \mathrm{mg} / \mathrm{dL}^{.17)}$

\section{Data Collection}

A self-administrated health questionnaire was used to collect information on age, sex, pharmacologic treatment for diabetes mellitus and hypertension, alcohol consumption, smoking status, aerobic exercise, family income, and education level. Anthropometric variables such as height $(\mathrm{cm})$, weight $(\mathrm{kg})$, and waist circumference $(\mathrm{WC}, \mathrm{cm})$ were measured by a trained health technician following the standardized procedure. BMI was calculated as weight in $\mathrm{kg}$ divided by height in meters squared $\left(\mathrm{m}^{2}\right)$. BP measurements were obtained from the right arm using a standard mercury sphygmomanometer (Baumanometer, 
Copiague, NY, USA). The average systolic BP (SBP) and diastolic BP (DBP) were obtained by calculating the mean of the second and third measurements after measuring the participant's BP 3 times at 5-minute intervals. Venous blood samples were obtained after an 8-hour minimum overnight fast. The level of fasting blood glucose (FBG) was measured using the Hitachi Automatic Analyzer 7600-210 (Hitachi, Tokyo, Japan). Glycated hemoglobin (HbAlc) values were measured using a G8 high-performance liquid chromatography analyzer (Tosoh, Tokyo, Japan).

\section{Assessment of Covariates}

For the purposes of calculating prevalence, diabetes mellitus was defined as the participant taking an antidiabetic medication, exhibiting an FBG level $\geq 126 \mathrm{mg} / \mathrm{dL}$, or exhibiting an HbAlc level $\geq 6.5 \%$. Hypertension was defined as the participant taking an antihypertensive medication, exhibiting a SBP level $\geq 140 \mathrm{~mm} \mathrm{Hg}$, or exhibiting a DBP level $\geq 140 \mathrm{~mm} \mathrm{Hg}$. Alcohol consumption was defined as self-reported consumption of alcohol in the previous year. Smoking status was classified as current smoker, ex-smoker, and non-smoker. Aerobic exercise was defined as the performance of $>150$ minutes of moderate intensity exercise or $>75$ minutes of high intensity exercise during the last week. Family income was divided into quartiles for analysis ( $<25$ th, 25-50th, $50-75$ th, and $>75$ th percentile values), while education level was divided into categories including: elementary school or less, graduated from middle school, graduated from high school, and graduated from college or higher.

\section{Statistical Analysis}

In KNHANES, the sampling results were weighted to provide a nationally representative prevalence estimate within the Korean population. The weights were calculated by accounting for the complex survey design, survey non-response, and post-stratification. The statistical analysis accounted for the complex sampling design of the KNHANES to minimize selection errors; the estimates reported in this study were obtained based on the primary sampling unit, stratification variables, and sampling weights. The analysis was adjusted for survey year to minimize the variations between survey years. ${ }^{18,19)}$

Statistical analysis was performed with IBM SPSS ver. 21.0 (IBM

Table 1. Baseline characteristics of the study population

\begin{tabular}{|c|c|c|c|c|}
\hline Characteristic & Total $(n=6,027)$ & Male $(n=2,934)$ & Female $(n=3,093)$ & P-value \\
\hline Age (v) & 47.5 & $42.6 \pm 0.4$ & $44.0 \pm 0.5$ & 0.003 \\
\hline BMI $\left(\mathrm{kg} / \mathrm{m}^{2}\right)$ & 23.9 & $24.4 \pm 0.1$ & $23.2 \pm 0.1$ & 0.000 \\
\hline Waist circumference (cm) & 82.4 & $85.5 \pm 0.2$ & $78.3 \pm 0.3$ & $<0.001$ \\
\hline Absolute grip strength $(\mathrm{kg})^{\star}$ & 30.5 & $39.5 \pm 0.2$ & $23.7 \pm 0.1$ & $<0.001$ \\
\hline Relative grip strength $(\mathrm{kg} / \mathrm{BMl})^{\dagger}$ & 1.2903 & $1.6351 \pm 0.0088$ & $1.0430 \pm 0.0070$ & $<0.001$ \\
\hline Total cholesterol (mg/dL) & 189.0 & $189.1 \pm 0.9$ & $189.8 \pm 0.8$ & 0.483 \\
\hline High-density lipoprotein cholesterol (mg/dL) & 49.9 & $46.9 \pm 0.3$ & $54.1 \pm 0.3$ & $<0.001$ \\
\hline Triglycerides (mg/dL) & 152.5 & $187.9 \pm 4.3$ & $120.3 \pm 2.3$ & $<0.001$ \\
\hline Low-density lipoprotein cholesterol (mg/dL) & 112.3 & $112.9 \pm 0.8$ & $112.4 \pm 0.6$ & 0.606 \\
\hline Medication for dyslipidemia & 9.3 & $5.3(0.4)$ & $8.6(0.5)$ & $<0.001$ \\
\hline Diabetes mellitus ${ }^{\ddagger}$ & 12.4 & $11.7(0.7)$ & $8.5(0.6)$ & $<0.001$ \\
\hline Hypertension ${ }^{\S}$ & 15.4 & $17.0(0.9)$ & $10.4(0.6)$ & $<0.001$ \\
\hline Alcohol consumptionll & 68.2 & $81.1(0.8)$ & $63.5(1.2)$ & $<0.001$ \\
\hline Smoking status & & & & $<0.001$ \\
\hline Current smoker & 18.2 & $36.7(1.1)$ & $4.9(0.5)$ & \\
\hline Ex-smoker & 20.4 & $32.8(1.0)$ & $5.4(0.5)$ & \\
\hline Non-smoker & 61.4 & $30.5(1.0)$ & $89.7(0.7)$ & \\
\hline Aerobic exercise" & 50.4 & $57.6(1.2)$ & $49.4(1.3)$ & $<0.001$ \\
\hline Family income (percentile) & & & & $<0.001$ \\
\hline$<25$ th & 17.0 & $11.9(0.8)$ & $16.0(1.1)$ & \\
\hline 25-50th & 24.8 & $24.0(1.2)$ & $23.8(1.2)$ & \\
\hline 50-75th & 28.4 & $31.6(1.4)$ & $29.9(1.4)$ & \\
\hline 75-100th & 29.8 & $32.5(1.5)$ & $30.3(1.7)$ & \\
\hline Education level & & & & $<0.001$ \\
\hline Elementary school or less & 26.2 & $15.7(0.8)$ & $23.6(1.1)$ & \\
\hline Middle school graduate & 13.0 & $10.9(0.7)$ & $12.4(0.7)$ & \\
\hline High school graduate & 31.3 & $36.9(1.2)$ & $32.2(1.1)$ & \\
\hline College graduate or higher & 29.5 & $36.5(1.3)$ & $31.7(1.4)$ & \\
\hline
\end{tabular}

Values are presented as mean \pm standard error for continuous variables using generalized linear models and \% (standard error) for categorical variables using Pearson's chisquare tests, unless otherwise stated.

BMl, body mass index.

${ }^{*}$ Average of three measurements of dominant hand. ${ }^{\dagger}$ Absolute hand grip strength divided by BMl. ${ }^{\ddagger}$ Took an antidiabetic medication, with a fasting blood glucose level $\geq 126$ $\mathrm{mg} / \mathrm{dL}$ or with a glycated hemoglobin level $\geq 6.5 \% .{ }^{{ }^{~}}$ Took an antihypertensive medication, with a systolic blood pressure level $\geq 140 \mathrm{~mm} \mathrm{Hg}$ or with a diastolic blood pressure level $\geq 140 \mathrm{~mm} \mathrm{Hg}$. "Had consumed alcohol in the past year. " $\mathrm{Had}>150$ minutes of moderate intensity exercise or $>75$ minutes of high intensity exercise in the previous week. 
Corp., Armonk, NY, USA). To compare groups within sex according to quartile of relative HGS, continuous variables were summarized as mean values with standard errors (SEs) using generalized linear models, and categorical variables were evaluated as percentages with SEs using Pearson's chi-square tests.

Because the baseline characteristics differed according to sex, the analyses were stratified by sex. To examine the relationship between relative HGS and dyslipidemia, we divided the participants into four groups according to the quartiles of relative HGS: first (Q1, $\leq 1.3427 \mathrm{~kg}$ / BMI), second (Q2, $1.3428 \mathrm{~kg} / \mathrm{BMI}-1.5772 \mathrm{~kg} / \mathrm{BMI})$, third (Q3, 1.5773 $\mathrm{kg} / \mathrm{BMI}-1.8194 \mathrm{~kg} / \mathrm{BMI})$, and fourth (Q4, $\geq 1.8195 \mathrm{~kg} / \mathrm{BMI})$ in men and first (Q1, $\leq 0.8406 \mathrm{~kg} / \mathrm{BMI}$ ), second (Q2, $0.8407 \mathrm{~kg} / \mathrm{BMI}-1.0134 \mathrm{~kg}$ / BMI), third (Q3, $1.0135 \mathrm{~kg} / \mathrm{BMI}-1.1852 \mathrm{~kg} / \mathrm{BMI}$ ), and fourth (Q4, $\geq 1.1853 \mathrm{~kg} / \mathrm{BMI}$ ) in women. For each sex, a multivariate adjusted logistic regression analysis was performed with three models to evaluate the relationship between relative HGS and dyslipidemia. The first model was unadjusted. The second model was adjusted for age. The third model was adjusted further for prevalence of diabetes mellitus, prevalence of hypertension, alcohol consumption, smoking status, aerobic exercise, family income, and education level. Odds ratios (ORs) and their corresponding 95\% confidence intervals (CIs) for each group were shown with reference to participants with highest quartile of relative HGS. A P-value of $<0.05$ was considered significant.

\section{RESULTS}

Altogether, 6,027 participants were included in the study. Baseline characteristics of the 2,934 men and 3,093 women are shown in Table 1. The mean age for men was 42.6 years and 44.0 years for women. The mean BMI for men was $24.4 \mathrm{~kg} / \mathrm{m}^{2}$ and $23.2 \mathrm{~kg} / \mathrm{m}^{2}$ for women. The mean absolute HGS for men was $39.5 \mathrm{~kg}$ and $23.7 \mathrm{~kg}$ for women; the corresponding mean relative HGS for men was $1.6351 \mathrm{~kg} / \mathrm{BMI}$ and $1.0430 \mathrm{~kg} / \mathrm{BMI}$ for women. There were significant differences in these characteristics between men and women.

Tables 2 and 3 present the association between relative HGS and clinical variables. Lower relative HGS was associated with cardiovascular risk in this study. The group with the highest relative HGS was younger, had lower rates of hypertension, and had higher HDL levels in both sexes and lower TG levels in women only. However, there was a positive relationship between relative HGS and alcohol consumption. Relative HGS was inversely associated with diabetes mellitus and menopause.

Table 2. General characteristics of men according to the quartiles of relative HGS

\begin{tabular}{|c|c|c|c|c|c|}
\hline \multirow{2}{*}{ Characteristic } & \multicolumn{4}{|c|}{ Relative HGS } & \multirow{2}{*}{ P-value } \\
\hline & Q1 & Q2 & Q3 & Q4 & \\
\hline No. of participants & 741 & 739 & 740 & 714 & \\
\hline Age (y) & $45.0 \pm 1.0$ & $45.8 \pm 0.7$ & $42.1 \pm 0.6$ & $38.7 \pm 0.5$ & $<0.001$ \\
\hline Total cholesterol (mg/dL) & $184.2 \pm 1.7$ & $190.5 \pm 1.6$ & $192.2 \pm 1.5$ & $188.1 \pm 1.7$ & 0.001 \\
\hline High-density lipoprotein cholesterol (mg/dL) & $45.3 \pm 0.5$ & $45.9 \pm 0.5$ & $46.7 \pm 0.5$ & $49.1 \pm 0.5$ & $<0.001$ \\
\hline Triglyceride (mg/dL) & $174.8 \pm 7.5$ & $198.9 \pm 7.9$ & $197.9 \pm 7.4$ & $177.6 \pm 6.9$ & 0.25 \\
\hline Low-density lipoprotein cholesterol (mg/dL) & $110.7 \pm 1.4$ & $114.2 \pm 1.5$ & $114.9 \pm 1.4$ & $111.2 \pm 1.5$ & 0.71 \\
\hline Medication for dyslipidemia & $7.2(1.0)$ & $8.5(1.1)$ & $4.5(0.8)$ & $2.3(0.6)$ & $<0.001$ \\
\hline Diabetes mellitus* & $17.0(1.5)$ & $13.2(1.4)$ & $11.8(1.4)$ & $6.9(1.1)$ & $<0.001$ \\
\hline Hypertension ${ }^{\dagger}$ & $17.7(1.7)$ & $20.6(1.9)$ & $19.6(1.9)$ & $11.1(1.3)$ & $<0.001$ \\
\hline Alcohol consumption ${ }^{\ddagger}$ & $62.4(2.2)$ & $83.1(1.5)$ & $84.8(1.3)$ & $87.9(1.2)$ & $<0.001$ \\
\hline Smoking status & & & & & $<0.001$ \\
\hline Current smoker & $23.1(1.9)$ & $33.0(2.0)$ & $40.3(2.1)$ & $45.8(2.2)$ & \\
\hline Ex-smoker & $36.7(2.1)$ & $37.9(2.1)$ & $30.7(1.9)$ & $27.0(1.7)$ & \\
\hline Non-smoker & $40.2(2.2)$ & $29.1(1.9)$ & $29.0(1.9)$ & $27.2(1.9)$ & \\
\hline Aerobic exercise ${ }^{\S}$ & $51.9(2.5)$ & $55.4(2.4)$ & $60.7(2.1)$ & $60.3(2.1)$ & 0.20 \\
\hline Family income (percentile) & & & & & $<0.001$ \\
\hline$<25$ th & $19.7(2.0)$ & $11.1(1.2)$ & $10.8(1.4)$ & $8.8(1.5)$ & \\
\hline 25-50th & $25.9(2.0)$ & $27.2(2.2)$ & $19.9(1.9)$ & $24.2(2.0)$ & \\
\hline 50-75th & $25.9(2.3)$ & $29.7(2.4)$ & $33.9(2.2)$ & $34.6(2.2)$ & \\
\hline 75-100th & $28.5(2.0)$ & $32.0(2.6)$ & $35.5(2.4)$ & $32.5(2.3)$ & \\
\hline Education level & & & & & $<0.001$ \\
\hline Elementary school or less & $36.4(2.3)$ & $16.3(1.5)$ & $11.2(1.4)$ & $5.4(0.9)$ & \\
\hline Middle school graduate & $11.5(1.3)$ & $11.7(1.3)$ & $10.2(1.3)$ & $10.5(1.3)$ & \\
\hline High school graduate & $27.8(2.1)$ & $34.2(2.2)$ & $39.6(2.3)$ & $44.2(2.3)$ & \\
\hline College graduate or higher & $24.3(2.2)$ & $37.8(2.4)$ & $29.0(2.2)$ & $39.9(2.4)$ & \\
\hline
\end{tabular}

Values are presented as mean \pm standard error for continuous variables using generalized linear models and \% (standard error) for categorical variables using Pearson's chisquare tests, unless otherwise stated.

HGS, handgrip strength.

${ }^{*}$ Took an antidiabetic medication, with a fasting blood glucose level $\geq 126 \mathrm{mg} / \mathrm{dL}$ or with a glycated hemoglobin level $\geq 6.5 \%$. ${ }^{\dagger}$ Took an antihypertensive medication, with a systolic blood pressure level $\geq 140 \mathrm{~mm} \mathrm{Hg}$ or with diastolic blood pressure level $\geq 140 \mathrm{~mm} \mathrm{Hg}$. ${ }^{\ddagger} H a d$ consumed alcohol in the past year. ${ }^{\S} \mathrm{Had}>150 \mathrm{minutes}$ of moderate intensity exercise, or $>75$ minutes of high intensity exercise in the previous week. 
Table 3. General characteristics of women according to the quartiles of relative HGS

\begin{tabular}{|c|c|c|c|c|c|}
\hline \multirow{2}{*}{ Characteristic } & \multicolumn{4}{|c|}{ Relative HGS } & \multirow{2}{*}{ P-value } \\
\hline & Q1 & Q2 & Q3 & Q4 & \\
\hline No. of participants & 774 & 773 & 773 & 773 & \\
\hline Age (y) & $52.4 \pm 1.0$ & $45.4 \pm 0.9$ & $42.9 \pm 0.7$ & $37.4 \pm 0.6$ & $<0.001$ \\
\hline Total cholesterol (mg/dL) & $192.6 \pm 1.6$ & $194.4 \pm 1.6$ & $189.9 \pm 1.4$ & $183.7 \pm 1.2$ & $<0.001$ \\
\hline High-density lipoprotein cholesterol (mg/dL) & $49.5 \pm 0.5$ & $52.6 \pm 0.6$ & $55.1 \pm 0.5$ & $57.9 \pm 0.6$ & $<0.001$ \\
\hline Triglycerides (mg/dL) & $139.6 \pm 3.8$ & $134.8 \pm 4.3$ & $116.0 \pm 3.8$ & $97.2 \pm 3.2$ & $<0.001$ \\
\hline Low-density lipoprotein cholesterol (mg/dL) & $117.4 \pm 1.3$ & $116.8 \pm 1.3$ & $111.8 \pm 1.1$ & $105.6 \pm 1.1$ & $<0.001$ \\
\hline Medication for dyslipidemia & $14.4(1.3)$ & $11.7(1.2)$ & $7.0(1.0)$ & $3.0(0.6)$ & $<0.001$ \\
\hline Diabetes mellitus* & $17.6(1.7)$ & $10.6(1.2)$ & $5.7(0.9)$ & $2.5(0.5)$ & $<0.001$ \\
\hline Hypertension $^{\dagger}$ & $17.5(1.7)$ & $12.5(1.4)$ & $8.2(1.0)$ & $5.4(0.8)$ & $<0.001$ \\
\hline Alcohol consumption ${ }^{\ddagger}$ & $47.6(2.2)$ & $61.4(2.2)$ & $69.9(2.1)$ & $71.2(2.0)$ & $<0.001$ \\
\hline Smoking status & & & & & 0.391 \\
\hline Current smoker & $3.4(0.8)$ & $5.4(1.2)$ & $5.1(1.0)$ & $5.5(1.1)$ & \\
\hline Ex-smoker & $5.1(1.0)$ & $4.7(0.9)$ & $4.8(0.9)$ & $6.8(1.1)$ & \\
\hline Non-smoker & $91.5(1.2)$ & $89.9(1.4)$ & $90.1(1.2)$ & $87.7(1.4)$ & \\
\hline Aerobic exercise ${ }^{\S}$ & $40.0(2.3)$ & $45.5(2.6)$ & $51.5(2.3)$ & $57.5(2.0)$ & $<0.001$ \\
\hline Family income (percentile) & & & & & $<0.001$ \\
\hline$<25$ th & $29.6(2.1)$ & $15.9(1.6)$ & $11.4(1.6)$ & $10.0(1.3)$ & \\
\hline 25-50th & $22.9(1.9)$ & $27.4(2.2)$ & $24.1(1.8)$ & $21.4(1.9)$ & \\
\hline 50-75th & $26.2(2.1)$ & $31.0(2.2)$ & $30.3(2.3)$ & $31.1(2.2)$ & \\
\hline 75-100th & $21.4(2.2)$ & $25.7(2.3)$ & $34.2(2.3)$ & $37.5(2.4)$ & \\
\hline Education level & & & & & $<0.001$ \\
\hline Elementary school or less & $50.0(2.4)$ & $28.8(2.0)$ & $14.4(1.6)$ & $7.9(1.1)$ & \\
\hline Middle school graduate & $13.0(1.4)$ & $12.5(1.4)$ & $12.9(1.4)$ & $11.6(1.4)$ & \\
\hline High school graduate & $16.9(1.8)$ & $33.1(2.0)$ & $36.2(2.0)$ & $29.0(2.1)$ & \\
\hline College graduate or higher & $20.0(2.1)$ & $25.6(2.2)$ & $36.5(2.4)$ & $41.4(2.3)$ & \\
\hline Menopause & $62.2(2.5)$ & $46.3(2.6)$ & $34.2(2.0)$ & $19.8(1.6)$ & $<0.001$ \\
\hline
\end{tabular}

Values are presented as mean \pm standard error for continuous variables using generalized linear models and \% (standard error) for categorical variables using Pearson's chisquare tests, unless otherwise stated.

HGS, handgrip strength.

${ }^{*}$ Took an antidiabetic medication, with a fasting blood glucose level $\geq 126 \mathrm{mg} / \mathrm{dL}$ or with a glycated hemoglobin level $\geq 6.5 \%$. ${ }^{\top}$ Took an antihypertensive medication, with a systolic blood pressure level $\geq 140 \mathrm{~mm} \mathrm{Hg}$ or with diastolic blood pressure level $\geq 140 \mathrm{~mm} \mathrm{Hg}$. ${ }^{\ddagger}$ Had consumed alcohol in the past year. ${ }^{8}$ Had $>150$ minutes of moderate intensity exercise, or $>75$ minutes of high intensity exercise in the previous week.

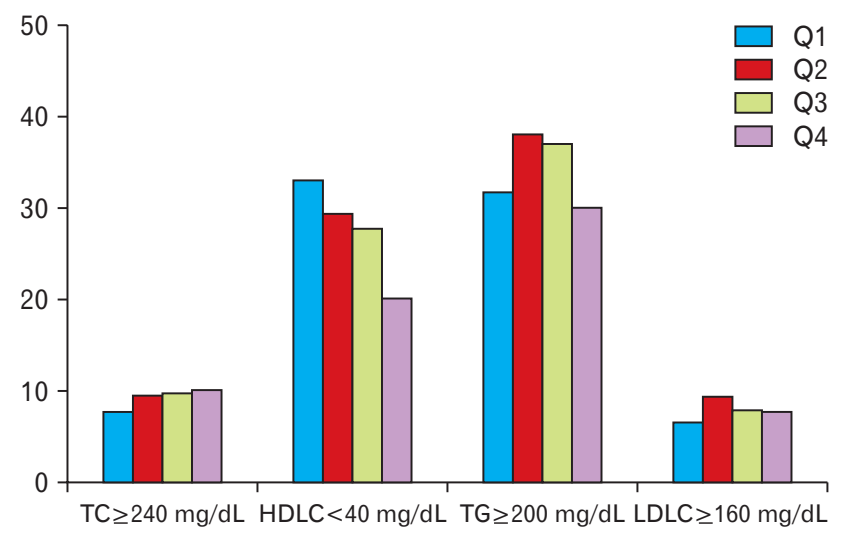

Figure 1. Components of dyslipidemia by quartile of relative handgrip strength in men. TC, total cholesterol; HDLC, high-density lipoprotein cholesterol; TG, triglyceride; LDLC, low-density lipoprotein cholesterol.

The categories of dyslipidemia by quartiles of relative HGS are shown in Figures 1 and 2 . We observed a statistically significant relationship between lipid profiles and quartiles of relative HGS. Among men, a higher relative HGS was associated with lower risk of low

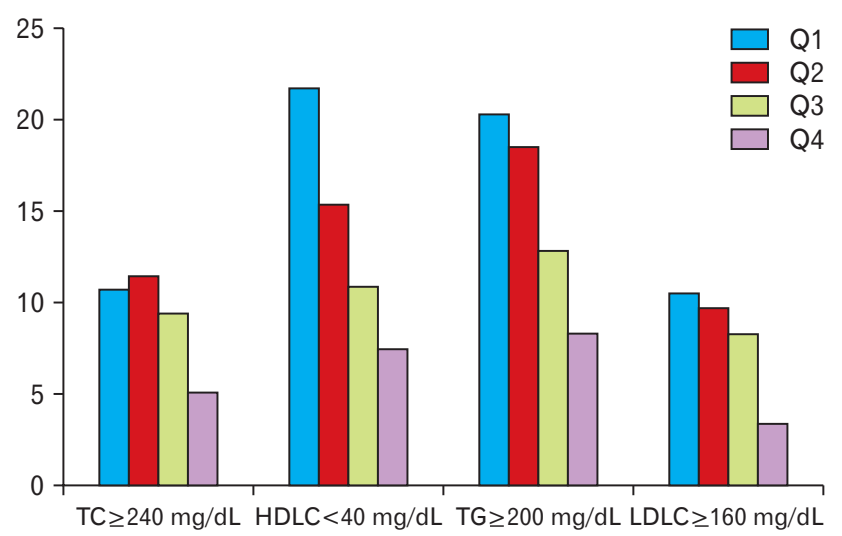

Figure 2. Components of dyslipidemia by quartile of relative handgrip strength in women. TC, total cholesterol; HDLC, high-density lipoprotein cholesterol; TG, triglyceride; LDLC, low-density lipoprotein cholesterol.

HDLC ( $<<0.001 ; \mathrm{Q} 1,33.1 \%$; Q2, 29.5\%; Q3, 27.8\%; and Q4, 20.3\%). Among women, a higher relative HGS was associated with lower risk of low HDLC ( $\mathrm{P}<0.001$; Q1, 21.7\%; Q2, 15.4\%; Q3, 10.9\%; and Q4, 7.5\%), high TG ( $\mathrm{P}<0.001$; $\mathrm{Q} 1,20.3 \%$; 2 , 18.5\%; $\mathrm{Q} 3,12.8 \%$; and $\mathrm{Q} 4,8.3 \%)$, and 
high LDLC ( $<<0.001$; Q1, 10.5\%; Q2, 9.7\%; Q3, 8.3\%; and Q4, 3.3\%).

In men, the unadjusted ORs for dyslipidemia were significantly higher among those in the lower relative HGS quartile groups than among those in the highest quartile group. There was a significantly negative correlation between relative HGS and dyslipidemia in women. After adjusting for age, prevalence of diabetes mellitus, prevalence of hypertension, alcohol consumption, smoking status, aerobic exercise, family income, and education level, relative HGS was inversely related to dyslipidemia in both men and women. The multivariableadjusted ORs for dyslipidemia in Q1, Q2, and Q3 relative to quartile 4 were $1.36,1.29$, and 1.23 in men, and $1.81,1.81$, and 1.39 in women, respectively (Table 4$)$.

\section{DISCUSSION}

This study examined the relationship between relative HGS and dyslipidemia using a nationwide survey of Korean adults. The results showed that there was a significant inverse association between relative HGS and dyslipidemia in both men and women. In this study, relative HGS was significantly inversely related to cardiovascular risk factors, particularly low HDL, aging, and hypertension. The prevalence of hypertension was higher in the group with lower relative HGS than in the group with the highest relative HGS. Diabetes mellitus also exhibited a negative relationship with relative HGS. The prevalence of diabetes was higher in the lowest group than in the highest group.

In a study of 350 schoolchildren, improved muscle strength has been shown to reduce cardiometabolic risk. ORs (95\% CIs) for borderline/low HDLC in the low, moderate, and high grip strength group were $1.00,0.58(0.35-0.98)$, and $0.28(0.16-0.48)$, respectively. ORs (95\% CIs) for borderline/high TGs in the low, moderate, and high grip strength group were 1.00, 0.79 (0.44-1.40), and 0.47 (0.25-0.88), respectively. After adjustment for BMI, grip strength was no longer associated with HDLC and TG levels. ${ }^{20)}$ In contrast, our study used relative HGS, a BMI-adjusted index, and the results were statistically signifi- cant. Some previous studies reported that absolute HGS was associated with metabolic syndrome, including abnormal lipid metabolism. A previous study conducted in UK adults demonstrated that lower HGS was associated with higher TG levels, BP, and WC, but not with lower HDLC and higher FBG levels. ${ }^{9}$ In a study of Japanese adults aged 65 years and older, a correlation was observed between HGS and metabolic syndrome. In the study's analysis of HGS and each component of the metabolic syndrome, abdominal obesity was a primary contributor to the occurrence of metabolic syndrome, while the other components (high TG levels, low HDLC levels, high FPG levels, and high BP) did not contribute to the occurrence of this condition. ${ }^{21)}$ In summary, the reported relationship between absolute HGS and lipid profiles differed between studies.

Recent studies investigated the association between relative HGS and metabolic syndrome. A study conducted among American adults showed that higher relative HGS was significantly associated with favorable HDLC, TG, FBG, and SBP levels, but not with LDLC level. ${ }^{14)}$ In China, using multivariable regression, relative HGS showed a favorable association with lipid metabolism (all $\mathrm{P}<0.001$; LDLC: $\mathrm{b}=-0.14$ [men], -0.19 [women]; TC: $\mathrm{b}=-0.20$ [men], -0.19 [women]; TG: $\mathrm{b}=-0.58$ [men], -0.55 [women]; HDLC: $\mathrm{b}=0.19$ [men], 0.22 [women]). ${ }^{22)}$ There were inverse relationships between relative HGS and lipid profile in American and Chinese adults, and our study found similar results. Particularly favorable results for the relationship between relative HGS and HDL have been observed in all studies.

It is noteworthy that our findings indicate an inverse relationship between grip strength and dyslipidemia. In men, there was an inverse relationship between relative HGS and low HDL. In women, there was a negative association between relative HGS and low HDL, high TG, and high LDL. After adjusting for various covariates, we found that low grip strength and dyslipidemia were significantly correlated in women, and the same tendency was observed in men. However, further studies are needed to determine the effect of muscle strength on blood cholesterol and TG. The development of dyslipidemia is known to be

Table 4. Association between relative handgrip strength and dyslipidemia

\begin{tabular}{|c|c|c|c|c|c|c|}
\hline & \multicolumn{2}{|c|}{ Model 1} & \multicolumn{2}{|c|}{ Model 2} & \multicolumn{2}{|c|}{ Model 3} \\
\hline & OR (95\% Cl) & P-value & $\mathrm{OR}(95 \% \mathrm{Cl})$ & P-value & OR (95\% Cl) & P-value \\
\hline Male & & 0.008 & & 0.080 & & 0.194 \\
\hline Q1 & $1.20(0.95-1.52)$ & & $1.05(0.83-1.35)$ & & $1.36(1.00-1.83)$ & \\
\hline Q2 & $1.46(1.15-1.85)$ & & $1.26(0.99-1.62)$ & & $1.29(0.98-1.70)$ & \\
\hline Q3 & $1.37(1.08-1.74)$ & & $1.29(1.01-1.64)$ & & $1.23(0.95-1.60)$ & \\
\hline Q4 & 1 (Reference) & & 1 (Reference) & & 1 (Reference) & \\
\hline Female & & $<0.001$ & & $<0.001$ & & 0.001 \\
\hline Q1 & $3.46(2.66-4.51)$ & & $2.12(1.59-2.84)$ & & $1.81(1.30-2.50)$ & \\
\hline Q2 & $2.75(2.09-3.63)$ & & $2.13(1.60-2.85)$ & & $1.81(1.32-2.47)$ & \\
\hline Q3 & $1.79(1.38-2.32)$ & & $1.50(1.16-1.94)$ & & $1.39(1.07-1.81)$ & \\
\hline Q4 & 1 (Reference) & & 1 (Reference) & & 1 (Reference) & \\
\hline
\end{tabular}

Values are presented as odd ratios (95\% confidence interval) from the logistic regression models. Model 1 was unadjusted. Model 2 was adjusted for age. Model 3 was adjusted for age, prevalence of diabetes mellitus, prevalence of hypertension, alcohol consumption, smoking status, aerobic exercise, family income, and education level. Additionally, model 3 in women was adjusted for menopause.

$\mathrm{OR}$, odd ratio; $\mathrm{Cl}$, confidence interval. 
influenced by many factors, including obesity, alcohol consumption, high carbohydrate diet, estrogen, and heredity. When considering these factors, the difference between men and women in this study can be explained by differences in sex hormones ${ }^{23,24)}$ and drinking behavior (Table 1).

The present study shows that the prevalence of hypertension and diabetes mellitus increased as relative HGS decreased in both men and women, which was consistent with the results of recently published studies. Muscle strength measured by grip strength was inversely related to FBG level, HbAlc level, insulin resistance, and type 2 diabetes mellitus in Korean adults. ${ }^{25)}$ In a large and nationally representative survey of adults with normal weight, grip strength was lower among individuals with both undiagnosed and diagnosed diabetes ( $\beta=-10.02, \mathrm{P}<0.0001$ for undiagnosed; $\beta=-8.21, \mathrm{P}=0.03$ for diagnosed) and hypertension ( $\beta=-6.6, \mathrm{P}=0.004$ for undiagnosed; $\beta=-4.27, \mathrm{P}=0.04$ for diagnosed) than among those without diabetes mellitus and hypertension after adjustment for age, sex, race, smoking status, and presence of a first-degree relative with the disease. ${ }^{26)}$

Despite the significant findings for grip strength and cardiometabolic risk factors, the percentage of men who were current smokers increased as grip strength increased, although this relationship was not observed among women. These findings could be attributed to other factors, such as biochemical or anthropometric factors that might have affected grip more than smoking status. The proportion of current smokers was $36.7 \%$ in men and $4.9 \%$ in women. Most of the women included in the study were nonsmokers, and the prevalence of current smokers and ex-smokers was significantly lower in among women than among men.

Skeletal muscles play a central role in the regulation of whole body metabolism, including energy expenditure and glucose storage stimulated by insulin. ${ }^{27-29)}$ Because a decrease in muscle mass and contraction intensity reduces the area responsible for glucose transport and worsens insulin resistance, muscle is considered to be an important component of systemic insulin resistance. ${ }^{30)}$ In a healthy skeletal muscle, the rate of fatty acid (FA) uptake is equivalent to the rate of FA oxidation. Skeletal muscle dysfunction causes the rate of FA uptake to exceed the rate of FA oxidation, resulting in the deposition of lipid intermediates. This process is involved in the development of dyslipidemia by triggering an increase in TG and LDLC, and a decrease in HDLC, although muscle strength and muscle function are not equivalent.

Body weight, height, and body mass are known to affect grip strength. ${ }^{13)}$ As we have noted, the associations between grip strength and other characteristics have differed between studies, depending on whether the analyzed measures of grip strength controlled for BMI. In recent studies, relative HGS, adjusted for BMI, was inversely related to cardiometabolic risk. ${ }^{12,15,22)}$ The results of our study are consistent with this previous research. On the other hand, the relationship between absolute muscle strength and dyslipidemia remains controversial. ${ }^{12,20)}$ Although the results were not shown, absolute grip strength was not significantly associated with dyslipidemia in this study. There is no standardized method for evaluating grip strength. However, we have found that relative HGS is a more significant indicator than absolute grip strength when assessing cardiometabolic risk factors, including dyslipidemia.

This study had some limitations. Because the study was cross-sectional in nature, longitudinal analyses are still necessary to support conclusions about the effect of grip strength on cardiometabolic risk factors. Additionally, the evaluation of muscle strength was only based on the measurement of grip strength; greater muscle strength generally may not equate to improved muscular health or efficiency. Grip strength is also known to be greatly affected by age; although we adjusted for age in our analyses, it would also be useful to perform analyses that are stratified by age in future research. Because data regarding the ingredients of the drugs taken by participants were not obtained, we could not consider the effect of drugs such as statins on muscles. Finally, confounding factors such as a high carbohydrate diet and a family history of dyslipidemia were not considered.

This study also had several strengths. To consider the effect of BMI on grip strength, we used relative HGS. To our knowledge, this is the first study to examine associations between relative grip strength and dyslipidemia among a nationally representative sample from Korean adults who participated in the Korea National Health and Nutrition Examination Survey (KNHANES) VI 2014-2015. We also adjusted for variables, such as health behavior and socioeconomic level, that affect both grip strength and dyslipidemia, and our results suggest the possibility that relative HGS could be used as an indicator for dyslipidemia. Additionally, we suggest that increasing muscle strength could prevent dyslipidemia and cardiovascular disease.

In conclusion, our findings suggest that greater relative HGS is associated with healthier HDLC concentrations in Korean adults. While longitudinal analyses are warranted to determine whether grip strength is an independent predictor of dyslipidemia, musclestrengthening exercise should nevertheless be considered for enhancing health outcomes.

\section{CONFLICT OF INTEREST}

No potential conflict of interest relevant to this article was reported.

\section{ORCID}

Bo Mi Kim: https://orcid.org/0000-0002-8897-3557 Yu Hyeon Yi: https://orcid.org/0000-0002-1786-2737 Yun Jin Kim: https://orcid.org/0000-0002-0204-3253 Sang Yeoup Lee: https://orcid.org/0000-0002-3585-9910 Jeong Gyu Lee: https://orcid.org/0000-0001-7160-0714 Young Hye Cho: https://orcid.org/0000-0003-2176-6227 Young Jin Tak: https://orcid.org/0000-0002-4645-5866 Hye Rim Hwang: https://orcid.org/0000-0001-7658-3749 Seung Hun Lee: https://orcid.org/0000-0002-0976-8708 Eun Ju Park: https://orcid.org/0000-0003-2415-8243 Youngin Lee: https://orcid.org/0000-0003-0141-7484 


\section{REFERENCES}

1. World Health Organization. Status of the health-related SDGs. In: World Health Organization, editor. World health statistics 2018: monitoring health for the sdgs, sustainable development goals. Geneva: World Health Organization; 2018. p. 4-7.

2. Shin HY, Lee JY, Kim JE, Lee S, Youn H, Kim H, et al. Cause-of-death statistics in 2016 in the Republic of Korea. J Korean Med Assoc 2018; 61:573-84.

3. Kim HC. Epidemiology of dyslipidemia in Korea. J Korean Med Assoc 2016;59:352-7.

4. Jee SH, Jang Y, Oh DJ, Oh BH, Lee SH, Park SW, et al. A coronary heart disease prediction model: the Korean Heart Study. BMJ Open 2014;4: e005025.

5. Legrand D, Adriaensen W, Vaes B, Mathei C, Wallemacq P, Degryse J. The relationship between grip strength and muscle mass (MM), inflammatory biomarkers and physical performance in communitydwelling very old persons. Arch Gerontol Geriatr 2013;57:345-51.

6. Wind AE, Takken T, Helders PJ, Engelbert RH. Is grip strength a predictor for total muscle strength in healthy children, adolescents, and young adults? Eur J Pediatr 2010;169:281-7.

7. Celis-Morales CA, Welsh P, Lyall DM, Steell L, Petermann F, Anderson J, et al. Associations of grip strength with cardiovascular, respiratory, and cancer outcomes and all cause mortality: prospective cohort study of half a million UK Biobank participants. BMJ 2018;361:k1651.

8. Gubelmann C, Vollenweider P, Marques-Vidal P. Association of grip strength with cardiovascular risk markers. Eur J Prev Cardiol 2017;24: 514-21.

9. Sayer AA, Syddall HE, Dennison EM, Martin HJ, Phillips DI, Cooper C, et al. Grip strength and the metabolic syndrome: findings from the Hertfordshire Cohort Study. QJM 2007;100:707-13.

10. Chin SO, Rhee SY, Chon S, Hwang YC, Jeong IK, Oh S, et al. Sarcopenia is independently associated with cardiovascular disease in older Korean adults: the Korea National Health and Nutrition Examination Survey (KNHANES) from 2009. PLoS One 2013;8:e60119.

11. Leong DP, Teo KK, Rangarajan S, Lopez-Jaramillo P, Avezum A Jr, Orlandini A, et al. Prognostic value of grip strength: findings from the Prospective Urban Rural Epidemiology (PURE) study. Lancet 2015; 386:266-73.

12. Yi DW, Khang AR, Lee HW, Son SM, Kang YH. Relative handgrip strength as a marker of metabolic syndrome: the Korea National Health and Nutrition Examination Survey (KNHANES) VI (20142015). Diabetes Metab Syndr Obes 2018;11:227-40.

13. Liao KH. Hand grip strength in low, medium, and high body mass index males and females. Middle East J Rehabil Health Stud 2016;3: e53229.

14. Lawman HG, Troiano RP, Perna FM, Wang CY, Fryar CD, Ogden CL. Associations of relative handgrip strength and cardiovascular disease biomarkers in U.S. adults, 2011-2012. Am J Prev Med 2016;50:677-83.

15. Lee WJ, Peng LN, Chiou ST, Chen LK. Relative handgrip strength is a simple indicator of cardiometabolic risk among middle-aged and older people: a nationwide population-based study in Taiwan. PLoS One 2016;11:e0160876.

16. Lee WJ, Peng LN, Chiou ST, Chen LK. Physical health indicators improve prediction of cardiovascular and all-cause mortality among middle-aged and older people: a national population-based study. Sci Rep 2017;7:40427.

17. Committee for the Korean Guidelines for the Management of Dyslipidemia. 2015 Korean guidelines for the management of dyslipidemia: executive summary (English translation). Korean Circ J 2016;46:275306.

18. Wang H, Jeong H, Kim NH, Kang Y, Hwang K, Lee H, et al. Association between beverage intake and obesity in children: the Korea National Health and Nutrition Examination Survey (KNHANES) 2013-2015. Nutr Res Pract 2018;12:307-14.

19. Kweon S, Kim Y, Jang MJ, Kim Y, Kim K, Choi S, et al. Data resource profile: the Korea National Health and Nutrition Examination Survey (KNHANES). Int J Epidemiol 2014;43:69-77.

20. Blakeley CE, van Rompay MI, Schultz NS, Sacheck JM. Relationship between muscle strength and dyslipidemia, serum 25(OH)D, and weight status among diverse schoolchildren: a cross-sectional analysis. BMC Pediatr 2018;18:23.

21. Ishii S, Tanaka T, Akishita M, Ouchi Y, Tuji T, Iijima K, et al. Metabolic syndrome, sarcopenia and role of sex and age: cross-sectional analysis of Kashiwa cohort study. PLoS One 2014;9:e112718.

22. Li D, Guo G, Xia L, Yang X, Zhang B, Liu F, et al. Relative handgrip strength is inversely associated with metabolic profile and metabolic disease in the general population in China. Front Physiol 2018;9:59.

23. Mendelsohn ME, Rosano GM. Hormonal regulation of normal vascular tone in males. Circ Res 2003;93:1142-5.

24. Granfone A, Campos H, McNamara JR, Schaefer MM, Lamon-Fava S, Ordovas JM, et al. Effects of estrogen replacement on plasma lipoproteins and apolipoproteins in postmenopausal, dyslipidemic women. Metabolism 1992;41:1193-8.

25. Lee MR, Jung SM, Bang H, Kim HS, Kim YB. Association between muscle strength and type 2 diabetes mellitus in adults in Korea: data from the Korea National Health and Nutrition Examination Survey (KNHANES) VI. Medicine (Baltimore) 2018;97:e10984.

26. Mainous AG 3rd, Tanner RJ, Anton SD, Jo A. Grip strength as a marker of hypertension and diabetes in healthy weight adults. Am J Prev Med 2015;49:850-8.

27. Turcotte LP, Fisher JS. Skeletal muscle insulin resistance: roles of fatty acid metabolism and exercise. Phys Ther 2008;88:1279-96.

28. Wolfe RR. The underappreciated role of muscle in health and disease. Am J Clin Nutr 2006;84:475-82.

29. Riechman SE, Lee CW, Chikani G, Chen VCW, Lee TV. Cholesterol and skeletal muscle health. World Rev Nutr Diet 2009;100:71-9.

30. Kalyani RR, Corriere M, Ferrucci L. Age-related and disease-related muscle loss: the effect of diabetes, obesity, and other diseases. Lancet Diabetes Endocrinol 2014;2:819-29. 